\title{
Knobloch syndrome associated with Polymicrogyria and early onset of retinal detachment: two case reports
}

\author{
Robert J. White, Yao Wang, Peter Tang and Sandra R. Montezuma*
}

\begin{abstract}
Background: Knobloch Syndrome (KS) is a rare congenital syndrome characterized by occipital skull defects and vitreoretinal degeneration. Retinal detachment (RD) often occurs at the end of the first decade of life or later. Aside from occipital skull defects, central nervous system abnormalities are uncommon.

Case presentations: We report on two siblings with KS. The first, a seven month old male, presented with nystagmus and was found to have a serous RD and a tessellated retinal appearance. His sister had a history of multiple visual abnormalities and had a similar retinal appearance although no signs of RD, but retina staphylomas. Genetic testing performed on both siblings showed a mutation in COL18A1, diagnostic of KS. MRI of both siblings demonstrated polymicrogyria but did not show occipital defects.

Conclusions: Although several families with KS have been described previously, our case is noteworthy for several reasons. The RD observed in our first patient occurred at an early age, and we find evidence of only one patient with KS who had an RD identified at an earlier age. The findings of polymicrogyria are not characteristic of KS, and we found only a few previous reports of this association. Additionally, we review potential treatment options for this condition.
\end{abstract}

Keywords: Knobloch syndrome, COL18A1, Retinal detachment, Polymicrogyria, Case report

\section{Background}

Knobloch Syndrome (KS) is a rare autosomal recessive syndrome first described in 1971 characterized by vitreoretinal degeneration and occipital skull abnormalities [1]. Clinical heterogeneity is present, although virtually all patients have ocular abnormalities that typically result in bilateral loss of vision. Ophthalmic findings include retinal detachment (RD), high myopia, early-onset cataracts, pigment dispersion, congenital glaucoma, and lens subluxation.

Midline occipital defects, namely bone defects, encephalocele, or aplasia cutis congenita, are characteristic findings. Other central nervous system findings are overall rare and not considered to be stereotypic features of KS. Caglayan et al. review seven cases of patients with KS associated with other central nervous system findings

* Correspondence: smontezu@umn.edu

Department of Ophthalmology and Visual Neurosciences, University of

Minnesota, 420 Delaware St. SE, MMC 493, Minneapolis, MN 55455-0501, USA including pachygyria, polymicrogyria and cerebellar atrophy among other findings [2]. Developmental delay is observed in only a minority of patients, although is observed more frequently in patients who also possess central nervous system abnormalities [2, 3]. Other less common findings include seizures, hyperextensibility of joints, lung hypoplasia, cardiac dextroversion, midface hypoplasia, flat nasal bridge, and duplicated renal collecting system observed in single families [4].

The causative gene in KS has been identified as COL18A1, which encodes for collagen type XVIII $\alpha-1$ chain. It is ubiquitously expressed in vascular and epithelial basement membranes and has multiple functions in ocular and neurologic development including maintenance of the basement membrane, cell proliferation, and angiogenesis [5].

Herein, we describe two siblings with KS associated with polymicrogyria, an anomaly sporadically associated with KS $[2,6]$. Polymicrogyria is a condition characterized by multiple small gyri leading to an abnormally 
thick cerebral cortex. It presents with variety of clinical symptoms dependent on the specific region of the brain that is affected, although seizures and developmental delay are commonly described. Our first case is also noteworthy as the patient presented with a RD at only 7-months-old, which to our knowledge is the second youngest age reported to date in a patient with KS [7]. We then briefly describe potentially promising treatment options for KS.

\section{Case presentations Case 1}

A 7-month-old Hispanic male was referred for nystagmus. He was born at term and exhibited normal growth but had motor and social developmental delay. Family history was significant for an older sister (Case 2) with visual abnormalities. His parents and two older brothers did not have any clinically significant visual problems. On examination, the patient fixed and followed with the left eye (OS) but not with the right eye (OD). Cycloplegic refraction $(\mathrm{CR})$ was found to be $-2.50+3.50 \times 090^{\circ}$ $\mathrm{OD}$ and $-7.00+3.50 \times 090^{\circ} \mathrm{OS}$. Anterior segment examination was unremarkable for both eyes (OU). Funduscopic examination OD showed a tilted optic nerve with trace pallor and a large posterior RD involving the macula with surrounding demarcation lines and a subretinal fibrotic band (Fig. 1a). The remainder of the retina appeared thin and atrophic. No retinal tears or holes were identified. Fundus examination OS showed a tilted optic nerve, a tessellated retinal appearance, retinal pigment epithelium (RPE) mottling, and central macular atrophy. There was no evidence of a retinal tear or detachment.

B-scan ultrasound OD confirmed a posterior RD (Fig. 1b). Fluorescein angiography (FA) OD demonstrated early and late hyperfluorescence consistent with pooling (Fig. 1c). FA OS only demonstrated RPE window defects and staining of drusen-like deposits. A full field electroretinogram (ERG) was performed according to ISCEV standards with the 2009 LKC machine and a small, infant Burian-Allen contact lens electrode under general anesthesia. This ERG showed moderately to severely depressed responses from both the cone and rod systems. The depressed responses were greater than the ones that could be attributed to anesthesia, myopic refractive error or partial retinal detachment (Fig. 2a). Optical coherence tomography (OCT) of OD showed an elevated retina with subretinal fluid; OS revealed RPE changes and thinning. A hereditary retinal dystrophy panel, covering roughly 180 genes, was significant for a mutation in the CNGB3 gene associated with achromatopsia. This, however, was inconsistent with the clinical presentation. Subsequent whole exome and mitochondrial DNA sequencing demonstrated a homozygous mutation in the
COL18A1 gene (NM_130445.3:c.2970_2971delAGinsC) associated with KS. Magnetic resonance imaging (MRI) of the brain demonstrated findings consistent with polymicrogyria but no evidence of an encephalocele (Fig. 3a). Fundus examination of the parents was unremarkable.

\section{Case 2}

This 13-year-old female was the sibling of the 7-monthold boy from Case 1. She had a past ocular history significant for an ERG demonstrating mild cone-rod dystrophy, bilateral macular chorioretinal staphylomas, subnormal visual acuity, high myopia, and strabismus status post extraocular muscle surgery OU. Her medical history was significant for a history of precocious $\mathrm{pu}$ berty at age 9 . Her vision had been poor since birth but remained stable at 20/200 OU for the past 8 years. She was being reevaluated in light of her brother's presentation. $\mathrm{CR}$ was found to be $-9.00+0.50 \times 110^{\circ} \mathrm{OD}$ and $-11.50+2.00 \times 080^{\circ} \mathrm{OS}$ with best-corrected visual acuity of 20/200 OU. On anterior segment exam there were bilateral patchy cortical cataracts. Fundus exam OU showed mild optic disc pallor, macular RPE atrophic changes, macular staphyloma, vascular attenuation, and a fundus with a tessellated appearance (Fig. 1d). Fundus autofluorescence confirmed significant RPE atrophic changes within the foveal and parafoveal regions of both eyes (Fig. 1e). OCT showed a mild staphyloma OD, moderate staphyloma OS, and irregular choriocapillaris with diffuse retinal thinning OU (Fig. 1f). A full field ERG was performed according to ISCEV standards using ESPION E3 system and DTL fiber recording electrodes. The ERG showed decreased amplitudes and delayed implicit times of the cone more than the rod system in both eyes. This ERG was consistent with cone-rod dystrophy that was stable compared to ERG obtained 4 years prior (Fig. 2b). Similar to her sibling from Case 1, the patient was found to have an identical homozygous mutation in the COL18A1 gene, and subsequent brain MRI showed findings consistent with polymicrogyria without evidence of an encephalocele (Fig. 3b).

\section{Discussion and conclusions}

In this paper, we report two siblings who presented with poor BCVA along with high myopia and anisometropia. Retinal examination and OCT demonstrated thinning of the RPE and an atrophic appearance, with a serous RD observed in one child although no leakage was seen on FA. ERG in both patients demonstrated significant depression of the cone and rod system. Whole genome and mitochondrial DNA sequencing eventually uncovered a mutation in homozygous mutation in the COL18A1 gene, diagnostic of KS. Notably, neither patient had the characteristic encephalocele and both had polymicrogyria demonstrated on MRI. 
a Fundus photos of Patient \#1

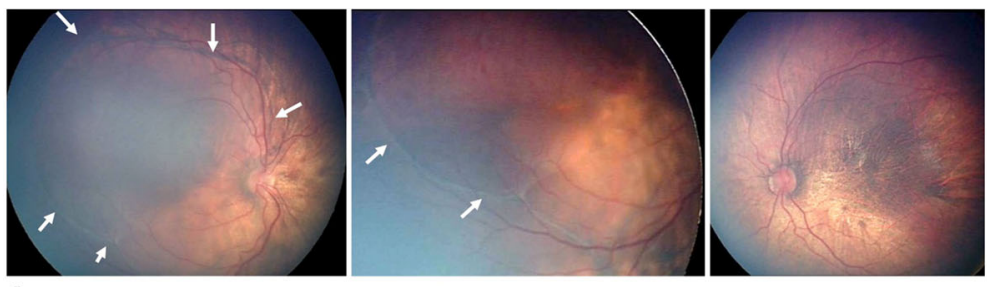

b B-scan US of right eye C FA of right eye

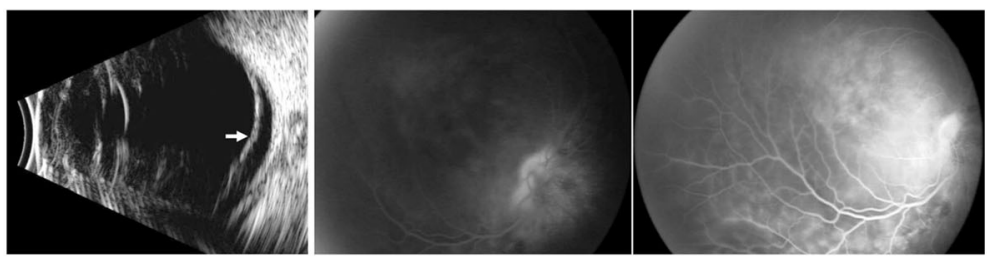

d Fundus photos of Patient \#2

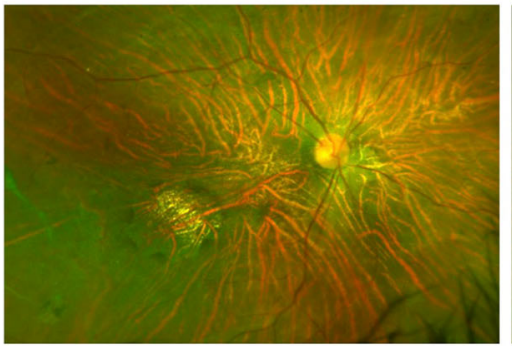

e $\mathrm{AF}$ of Patient \#2

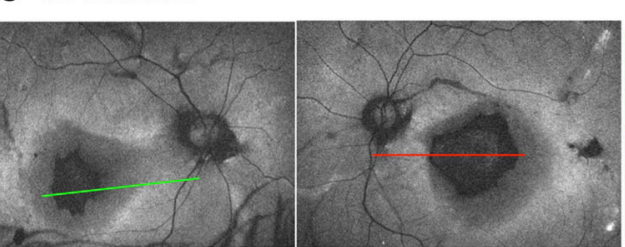

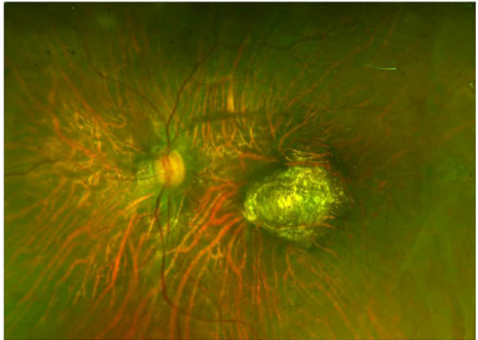

f Macula OCT of Patient \#2

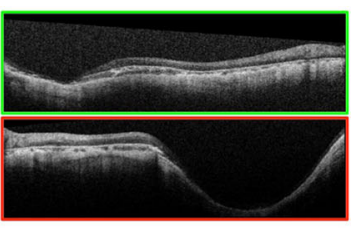

Fig. 1 a Fundus photo of the right eye (OD) of Patient 1 shows a tilted optic nerve with trace pallor and a large posterior serous retinal detachment (RD) of the macula with surrounding demarcation lines and a subretinal fibrotic band. The remainder of the retina appears thin and atrophic. Left eye (OS) shows a tilted optic nerve with pigment mottling and central macular atrophy but no evidence of a serous RD. b. B-scan of Patient 1 shows subretinal fluid OD. c Fluorescein angiography (FA) of Patient 1 shows posterior pooling with early and late optic nerve hyperfluorescence OD. d. Fundus exam of Patient 2 shows mild optic disc pallor, retinal pigment epithelial atrophy, mild staphyloma, vascular attenuation, and a fundus tigroidal appereance of both eyes (OU). e Fundus autofluorescence (FAF) of Patient 2 shows significant macular RPE atrophic changes OU with significant hypoautofluorescence within the fovea and parafoveal region. $\mathbf{f}$ Optical coherence tomography of Patient 2 showing a mild staphyloma OD, moderate staphyloma OS, and irregular choriocapillaris with diffuse retinal thinning OU

Our findings add to the literature supporting the spectrum of brain anomalies observed with KS, including polymicrogyria. Additionally, our cases are consistent with other reported cases of KS with polymicrogyria in which polymicrogyria did not occur with midline occipital defects $[2,6]$. Therefore, head imaging may be helpful in the diagnosis of KS and associated CNS abnormalities in patients with characteristic retinal findings but lacking an encephalocele. While the patient in Case 1 did experience delay in motor and social development, the patient in Case 2 experienced normal developmental milestones. To our knowledge, neither patient has any other neurologic abnormalities. In previously reported cases of KS with associated polymicrogyria, developmental delay was observed in most patients [2, 6].

The onset of RD at seven months of age in Case 1 was earlier than what is typically reported, as RDs tend to occur at the end of the first decade of life or later in patients with KS. There was one reported case of RD in the setting of KS identified at one month of age [7] and another case identified "before the age of one" [8]. KS is typically associated with rhegmatogenous $\mathrm{RD}$, consistent with the associated vitreoretinal degeneration, as opposed to the serous RD observed in our patient [9]. There is at least one prior case describing a serous RD occurring in a child with KS [10]. Unfortunately 


\section{a $\quad$ ERG of Patient \#1}
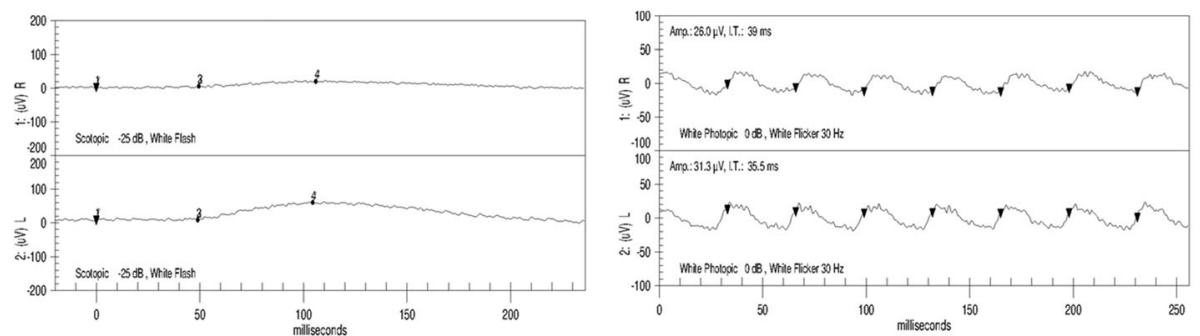

\section{b $\quad$ ERG of Patient \#2}
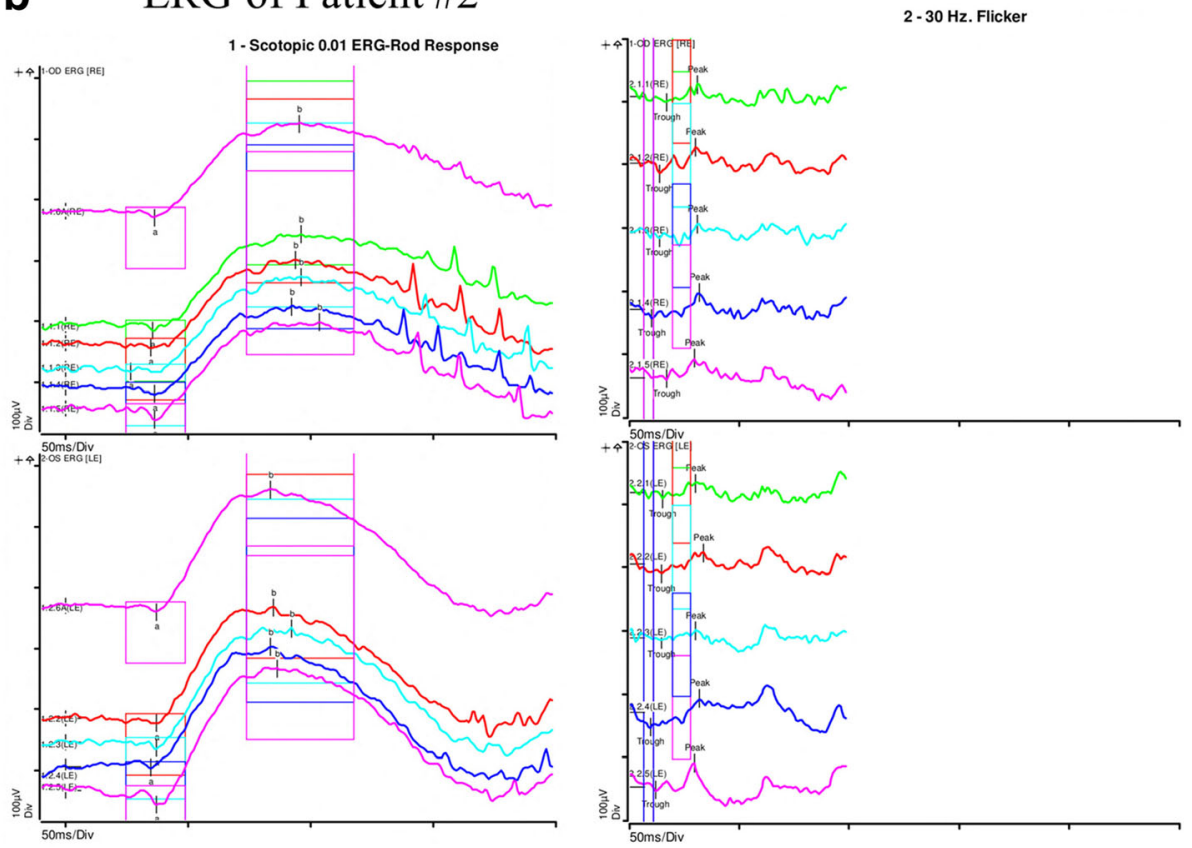

Fig. 2 Full field electroretinograms (ERG), performed according to ISCEV standards. a ERG of Patient 1 performed under general anesthesia shows moderate to severely depressed responses from both cone and rod systems that are greater than could be attributed to anesthesia, myopic refractive error, partial retinal detachment, or mild supraduction. b Full field ERG of Patient 2 shows decreased amplitudes and delayed implicit times of the cone more than the rod system of both eyes. This ERG is consistent with cone-rod dystrophy

however, the majority of case reports on patients with early onset of RD do not comment on the subtype of RD. $[6-8,11]$ The finding of a serous retinal detachment is of interest, as vitreoretinal degeneration would typically result in a rhegmatogenous RD. We find no basic science research to suggest a potential pathogenesis of serous RD development in patients with KS.

In the absence of obvious neurologic symptoms, the differential diagnosis of KS includes but is not limited to cone-rod dystrophy, Leber congenital amaurosis, retinitis pigmentosa, microcephaly lymphedema chorioretinal dysplasia syndrome, and Stickler syndrome. Khan et al. suggest that a triad of smooth iridies, ectopia lentis, and characteristic vitreoretinal degeneration is pathognomonic of KS based on an observation of eight children [10]. Notably, these findings were demonstrated in patients with an already known diagnosis of KS. We argue that the clinical triad described by Khan et al. is challenging to utilize within the clinical setting with an unknown diagnosis, and genetic testing is often essential for diagnosis. However, once a molecular diagnosis is reached, the patient should be reassessed to address possible associated ocular conditions of KS including pigment dispersion syndrome, $\mathrm{RD}$, lens subluxation and cataracts [6]. In addition, it is important to emphasize that the genetic testing results need to be correctly interpreted and correlate with the clinical findings to avoid misleading diagnosis, as in our first patient his initial retinopathy panel revealed a mutation in the CNGB3 gene associated with achromatopsia. The lack of correlation of this condition with his clinical findings led to additional genetic testing with subsequent whole exome and mitochondrial DNA sequencing demonstrating a mutation in the COL18A1. 


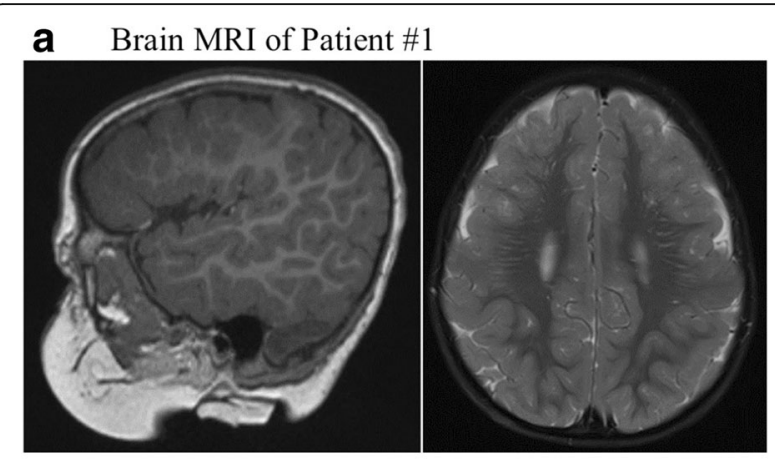

b Brain MRI of Patient \#2

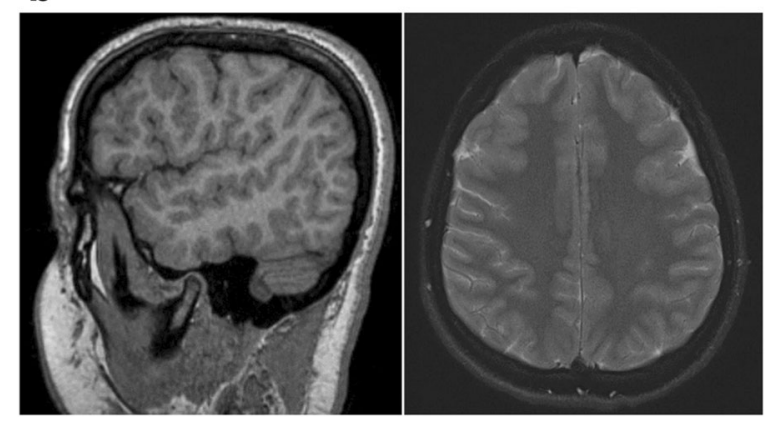

Fig. 3 Brain magnetic resonance imaging findings. a Sagittal $T_{1}$ weighted and axial $T_{2}$-weighted images of patient 1 demonstrating gray matter thickening in the frontal gyri bilaterally with scattered areas of increased $T_{2}$ signal intensity in the subcortical white matter consistent with polymicrogyria. There is no evidence of encephalocele. b Sagittal $T_{1}$-weighted and axial $T_{2}$-weighted images of patient 2 demonstrating gray matter thickening in the inferior and middle frontal gyri bilaterally consistent with polymicrogyria. There is no evidence of encephalocele

Although we contemplated repairing the serous RD in our patient, the prognosis for $\mathrm{KS}$ patients is often poor as could lead to the need for multiple interventions. Moysidis et al. describes a child with KS who underwent repair of a RD at 24 months of age and was also prophylactically treated with scleral buckle implantation [11]. Four years later, the patient is still doing well without evidence of recurrent RD, suggesting that this represents a potentially promising surgical prophylactic option. Given that these patients have high risk of RD during their life time, we offered to the parents treatment options of peripheral laser retinopexy with and without scleral buckle surgery vs cryopexy to the periphery. In Case 1, the parents elected for observation. They did however agree to have peripheral cryo-retinopexy OD only to prevent possible progression of the RD.

While treatment for KS is often supportive, recent advancements in our understanding of the pathophysiology of the disease come from studies in Drosophila [12]. Mutation of the COL18A1 gene resulted in mitochondrial structural disorganization that caused a decrease in energy generation and enhanced reactive oxygen species
(ROS) production. Interestingly, treating the mutants with the angiotensin II type 1 receptor antagonist losartan, a conventional hypertensive medication, has been shown to attenuate mitochondrial ROS production, improve mitochondrial morphology and restore function, suggesting a viable avenue for further investigation. Considerable research interest in the ocular reninangiotensin system and its role in disease may help guide future treatment options for patients with KS [13].

Further investigation is necessary to enhance our understanding of the pathophysiology of KS so that we may offer improved medical and surgical treatments for our patients.

\section{Abbreviations \\ CR: cycloplegic refraction; ERG: electroretinogram; KS: Knobloch Syndrome; MRI: magnetic resonance imaging; OCT: optical coherence tomography; OD: right eye; OS: left eye; OU: both eyes; RD: retinal detachment; \\ ROS: reactive oxygen species; RPE: retinal pigment epithelium}

\section{Acknowledgements}

We would like to thank Karol Rubin, Certified Genetic Counselor, for help with patient care and coordination.

\section{Funding}

Funds in support of our study to cover costs of publication fees come from the Lions Research Grant and an unrestricted grant from Research to Prevent Blindness.

\section{Availability of data and materials}

The data used and/or analyzed during the current study are available from the corresponding author on reasonable request.

\section{Authors' contributions}

All authors made substantial contributions to conception and design of the manuscript and were involved in drafting or revising the manuscript. All authors have approved the final version of the manuscript. All authors agree to be accountable for all aspects of the work.

\section{Ethics approval and consent to participate}

As this is a case report with no identifiable patient information, this report has been given exemption from requiring ethics approval by the University of Minnesota Internal Review Board.

\section{Consent for publication}

Written informed consent was obtained from the parents for the scientific use of medical records and imaging, in particular for publishing them and case information.

\section{Competing interest}

The authors declare that they have no competing interests.

\section{Publisher's Note}

Springer Nature remains neutral with regard to jurisdictional claims in published maps and institutional affiliations.

Received: 3 March 2017 Accepted: 19 November 2017

Published online: 25 November 2017

\section{References}

1. Knobloch WH, Layer JM. Retinal detachment and encephalocele. J Pediatr Ophthalmol Strabismus. 1971;8(3):181-4.

2. Caglayan AO, Baranoski JF, Aktar F, et al. Brain malformations associated with Knobloch syndrome-review of literature, expanding clinical spectrum, and identification of novel mutations. Pediatr Neurol. 2014;51(6):806-13.

3. Wenick AS, Barañano DE. Evaluation and management of pediatric rhegmatogenous retinal detachment. Saudi J Ophthalmol. 2012;26(3):255-63. 
4. Suzuki OT, Sertie AL, Der Kaloustian VM, et al. Molecular analysis of collagen $\mathrm{XVIII}$ reveals novel mutations, presence of a third isoform, and possible genetic heterogeneity in Knobloch syndrome. Am J Hum Genet. 2002;71(6):1320-9.

5. Seppinen L, Pihlajaniemi T. The multiple functions of collagen XVIII in development and disease. Matrix Biol. 2011;30(2):83-92.

6. Hull S, Arno G, CA K, et al. Molecular and clinical findings in patients with Knobloch syndrome. JAMA Ophthalmol. 2016;134(7):753-62.

7. Wilson C, Aftimos S, Pereira A, McKay R. Report of two sibs with Knobloch syndrome (encephalocoele and viteroretinal degeneration) and other anomalies. Am J Med Genet. 1998;78(3):286-90.

8. Duh EJ, Yao YG, Dagli M, Goldberg MF. Persistence of fetal vasculature in a patient with Knobloch syndrome: potential role for endostatin in fetal vascular remodeling of the eye. Ophthalmol. Retina. 2004;111(10):1885-8.

9. Richards AJ, Scott JD, Snead MP. Molecular genetics of rhegmatogenous retinal detachment. Eye. 2002;16(4):388-92.

10. Khan AO, Aldahmesh MA, Mohamed JY, Al-Mesfer S, Alkuraya FS. The distinct ophthalmic phenotype of Knobloch syndrome in children. Br J Ophthalmol. 2012;96(6):890-5.

11. Moysidis SN, Aziz HA, Rachitskaya AV, Berrocal AM. Prophylactic scleral buckle implantation in Knobloch syndrome. J Pediatr Ophthalmol Strabismus. 2014:51:e40-3.

12. Momota R, Narasaki M, Komiyama T, Naito I, Ninomiya Y, Ohtsuka A. Drosophila type XV/XVIII collagen mutants manifest integrin mediated mitochondrial dysfunction, which is improved by cyclosporin a and losartan. Int J Biochem Cell Biol. 2013:45(5):1003-11.

13. Giese MJ, Speth RC. The ocular renin-angiotensin system: a therapeutic target for the treatment of ocular disease. Pharmacol Ther. 2014;142(1):11-32.

\section{Submit your next manuscript to BioMed Central and we will help you at every step:}

- We accept pre-submission inquiries

- Our selector tool helps you to find the most relevant journal

- We provide round the clock customer support

- Convenient online submission

- Thorough peer review

- Inclusion in PubMed and all major indexing services

- Maximum visibility for your research

Submit your manuscript at www.biomedcentral.com/submit 